\title{
Adverse effects of interactions between antipsychotics and medications used in the treatment of cardiovascular disorders
}

\author{
Marcin Siwek $^{1}\left[\right.$ ] $\cdot$ Jarosław Woroń $^{2} \cdot$ Aleksandra Gorostowicz $^{3} \cdot$ Jerzy Wordliczek $^{4}$
}

Received: 19 September 2019 / Revised: 17 January 2020 / Accepted: 18 January 2020 / Published online: 2 March 2020

(c) The Author(s) 2020

\begin{abstract}
Background High level of comorbidity between bipolar disorder or schizophrenia and cardiovascular diseases (CVD) in clinical practice may contribute to drug-drug interactions between medications used in these conditions. The aim of this study was to evaluate harmful interactions between antipsychotics and medications used in treatment of CVD.

Methods The analysis of 52 cases of adverse reactions with a clinical picture indicates that they were the result of the combination of antipsychotic with cardiovascular medications.

Results The highest number of interactions with antipsychotics was recorded among beta-blockers ( $n=13,25 \%$ of all cases), including cardiac arrhythmias [atrial fibrillation $(n=1)$ : risperidone plus atenolol; bradycardia $(n=1)$ : perphenazine with metoprolol; ventricular arrhythmias: sertindole with metoprolol $(n=1)$ and ziprasidone with sotalol $(n=3)$ ] and hypotension [chlorprotixene with nebivolol or metoprolol $(n=2)$ ]. 12 cases concerned statins-myalgia, myopathy, or creatine kinase elevation appeared after combination of atorvastatin with haloperidol $(n=1)$, quetiapine $(n=3)$ or risperidone $(n=1)$, and simvastatin with quetiapine $(n=5)$ or risperidone $(n=2)$. There were also cases of interactions observed for the use of antipsychotics with anti-arrhythmic drugs (amiodarone, flecainide, propafenone) $(n=11)$, calcium channel blockers $(n=6)$, and other cardiac medications: clonidine, dabigatran, doxazosin, ivabradine, and losartan $(n=10)$.

Conclusions Due to a high risk of interactions and related adverse effects, particular attention should be paid while using cardiovascular medications with antipsychotics. Clinical decisions should be preceded by a detailed analysis of safety, riskbenefit ratio to search for, as safe as possible, drug combinations.
\end{abstract}

Keywords Antipsychotics $\cdot$ Cardiovascular disorders $\cdot$ Interactions $\cdot$ Adverse effects

\section{Introduction}

Cardiovascular disease (CVD) as well as psychiatric disorders and illnesses are, according to reports of the World Health Organization (WHO), one of the leading causes of

Marcin Siwek

drmarcinsiwek@gmail.com

1 Department of Affective Disorders, Chair of Psychiatry, Jagiellonian University Medical College, Kopernika st. 21 A, 31-501 Kraków, Poland

2 Department of Clinical Pharmacology, Chair of Pharmacology, Jagiellonian University Medical College, Kraków, Poland

3 Chair of Psychiatry, Jagiellonian University Medical College, Kraków, Poland

4 Intensive Care Interdisciplinary Clinic of the Jagiellonian University Medical College, Kraków, Poland disability in the world [1]. The relationship between CVD and psychiatric disorders is complex. People with bipolar disorder (BD) or schizophrenia (SZ) live 10-25 years shorter than the general population, and the most common cause of death in this group of patients is precisely CVD $[2,3]$.

In a meta-analysis from 2017 including 92 cross-sectional and longitudinal studies on the occurrence of CVD diseases in patients with serious mental illness (i.e., SZ, BD, or depression), approximately $10 \%$ of people in this population had at least one comorbid CVD disease [4]. The authors estimated that patients with mental illness had a 53\% increased risk of receiving a CVD diagnosis and an $85 \%$ increased risk of death due to CVD in comparison with the general population [4]. As important modulators, the authors recognized, among others, use of antipsychotics or increased body mass index. In another meta-analysis involving 13 cohort studies on CVD risk among people diagnosed with SZ (the analysis included a total of over 3.5 million people), it was shown 
that patients with this disease had a 1.53 times greater risk of CVD disease compared to control group [5]. A systematic review and meta-analysis of 31 cohort studies on mortality in patients with bipolar disorder showed that these patients were even twice as likely to die from CVD as compared to the general population [6]. In an epidemiological study involving over 40,000 people (with nearly 1500 people with a type $1 \mathrm{BD}$ ), the incidence of CVD diseases among patients diagnosed with bipolar disorder was almost fivefold higher than in the control group, even after taking into consideration factors such as age, obesity, and smoking [7]. On the other hand, in a Swedish study on over 6.5 million adult Swedes, including 6618 people with bipolar disorder, it was observed that patients with this diagnosis had an increased risk of developing CVD (1.3 times in women and 1.2 times in men) [8].

The use of polypharmacotherapy (defined as the use of several drugs at the same time- the lack of a unified nomenclature in the literature [9]) is a phenomenon that is frequent both in psychiatric [10] and cardiological patients [11]. Polytherapy increases the risk of potential adverse drug-drug interactions (DDI) of a pharmacokinetic or pharmacodynamic nature [10]. Due to the co-existence of psychiatric conditions described above (BD, SZ) and CVD in clinical practice, there may be an interaction between the drugs used in these conditions.

\section{Aim of the work}

The aim of this study was to assess the occurrence of adverse drug interactions between antipsychotics (used in the treatment of SZ and BD) and medicines used in the treatment of cardiovascular diseases. In all of the patients analyzed in our work, there were side effects whose clinical picture indicated (to a probable or certain degree) that side effects occurred in a combination of an antipsychotic drug with a drug used in CVD therapy.

\section{Methods}

Data regarding drug-related complications come from the material of the University Center for Clinical Drug Adverse Effects Monitoring and Study at the Clinic of Pharmacology of the Jagiellonian University Medical College in Krakow, which as a regional center monitors and reports, in accordance with existing legal acts, the complications of pharmacotherapy. The center also provides specialist consultations in the field of pharmacotherapy, adverse reactions, and drug interactions for outpatient clinics and hospitals from the following provinces: Małopolskie, Świętokrzyskie, Podkarpackie, and Ślaskie. Such consultations are carried out by specialists in the field of pharmacology, clinical pharmacology, internal diseases, and annually, their number varies between 850 and 1100 . Due to the constantly increasing number of complications of the interaction of psychotropic drugs with other simultaneously used drugs, the center regularly cooperates with the Department of Affective Disorders of the Department of Psychiatry, Jagiellonian University Medical College.

The data presented below were collected by analyzing orders for pharmacotherapy from persons in outpatient treatment (in geriatric, cardiac, and mental health clinics) and stationary treatment in emergency (SOR), internists, and cardiology departments. Data from the period from January 1, 2017 to March 30, 2018 were analyzed. To determine the correlation between the pharmacological treatment used and the adverse effects noted, pharmaco-epdemiological analysis was performed and the cause-effect relationship between the treatment and the clinical picture of the complications was determined. Pharmacodynamic and pharmacokinetic interactions as well as interactions related to the aggregation of the side effects of concurrently used antipsychotic drugs with cardiac drugs have been evaluated. In the analyzed cases, the average number of drugs used in the patient was 6 (min.: 4, max.: 9). In all cases, there was a cause-effect relationship probable $(n=41)$ or certain $(n=11)$ between joining the pharmacological treatment of cardiac drugs and the occurrence of complications with the clinical picture characteristic of the used drugs. The average age in the whole group of patients was $63.13(\mathrm{SD}=7.07)$.

\section{Interactions in the antipsychotic group $(n=52)$}

In the area of antipsychotic drugs, the interactions resulting from the administration of 13 of them were found in the analyzed cases. Table 1 provides information on the interactions that have occurred as a consequence of the combination of antipsychotic drugs with CVD drugs.

\section{Results}

The highest number of interactions between cardiac drugs and antipsychotics in the analyzed group was observed among beta-blockers-atenolol, nebivolol, metoprolol, and sotalol ( $n=13,25 \%$ of cases). The most frequent adverse reaction in this subgroup was cardiac arrhythmias ( $n=6$, approximately $11.5 \%$ of cases) of atrial fibrillation (risperidone with atenolol, $n=1)$, bradycardia $(n=1$, perphenazine with metoprolol), or ventricular arrhythmias [in case of the connections of sertindole with metoprolol $(n=1)$ and ziprasidone with sotalol $(n=3)]$. In one case, ventricular arrhythmias and death occurred in which the interaction between ziprasidone and satolol occurred. In two cases, hypotension was indicated due to the combination of chlorprotixene with nebivolol or metoprolol. One 


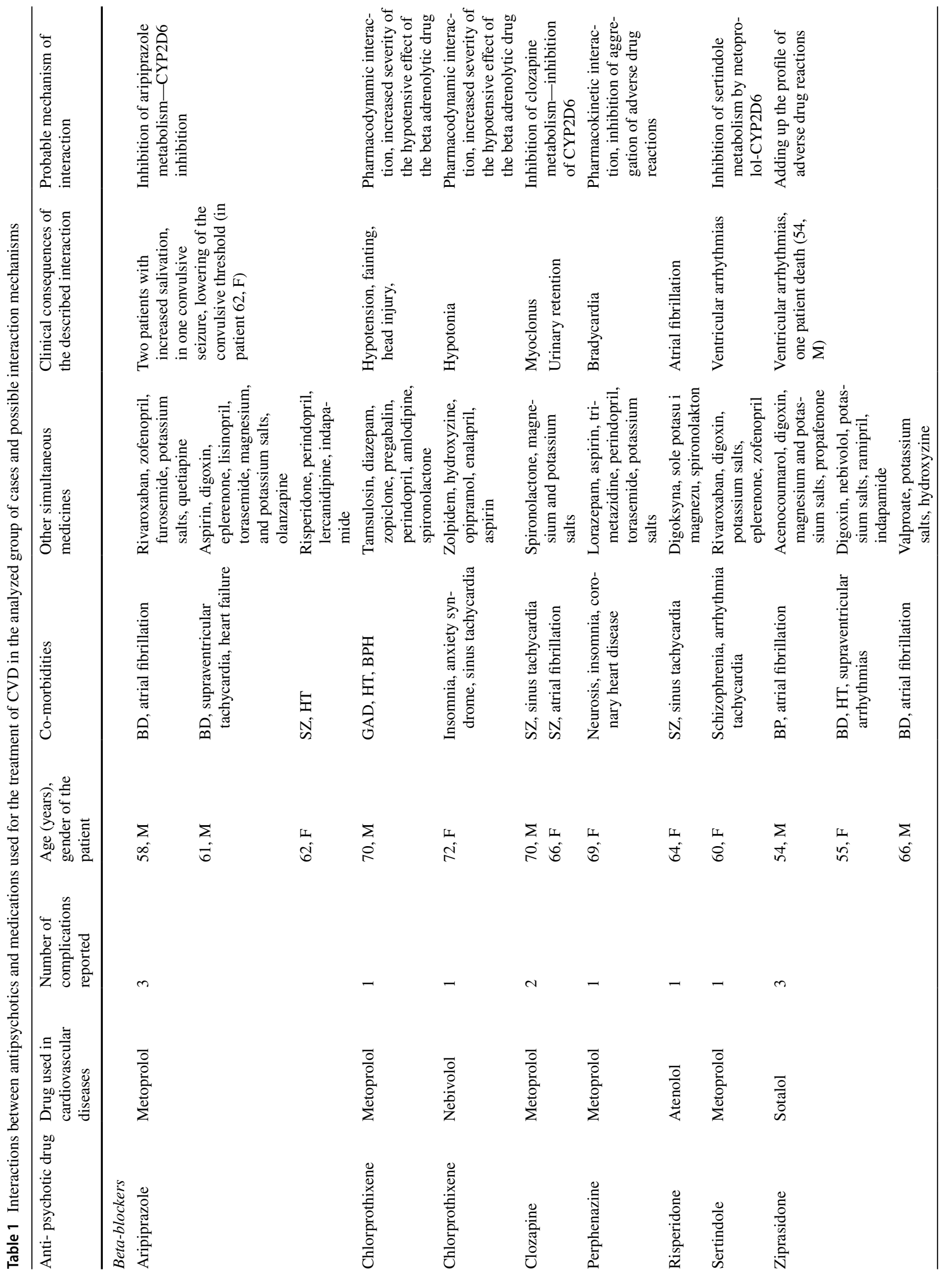




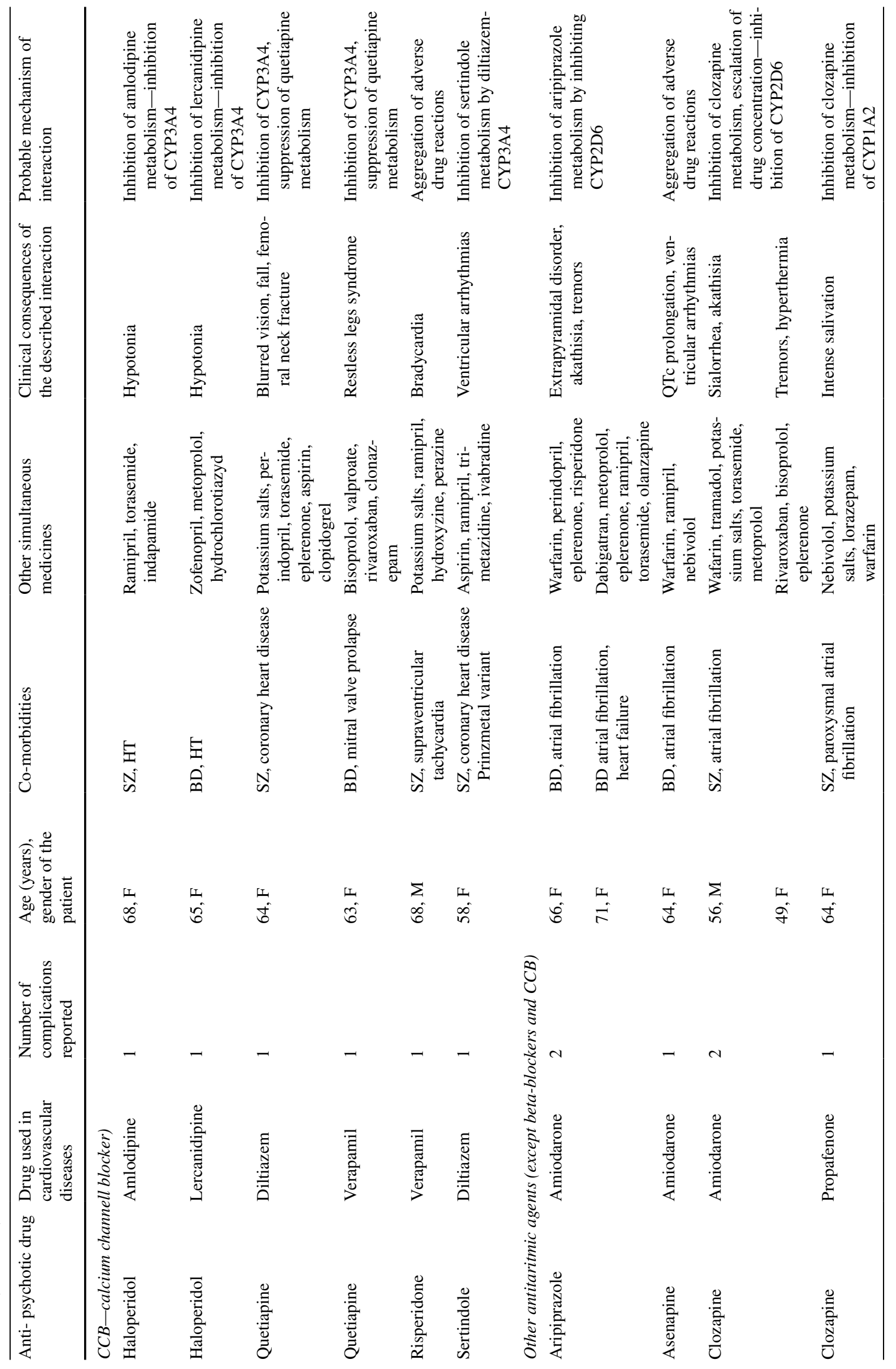




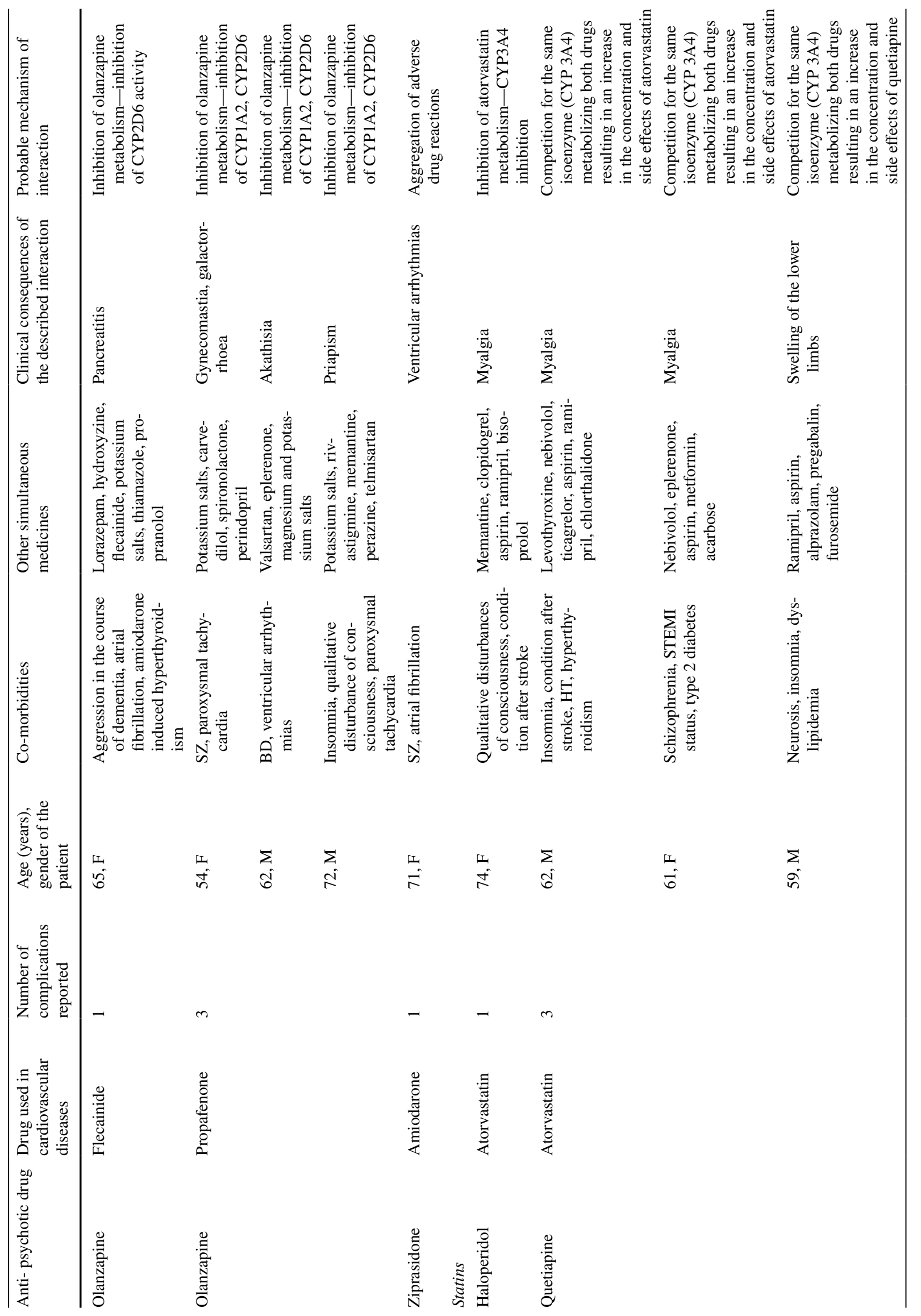




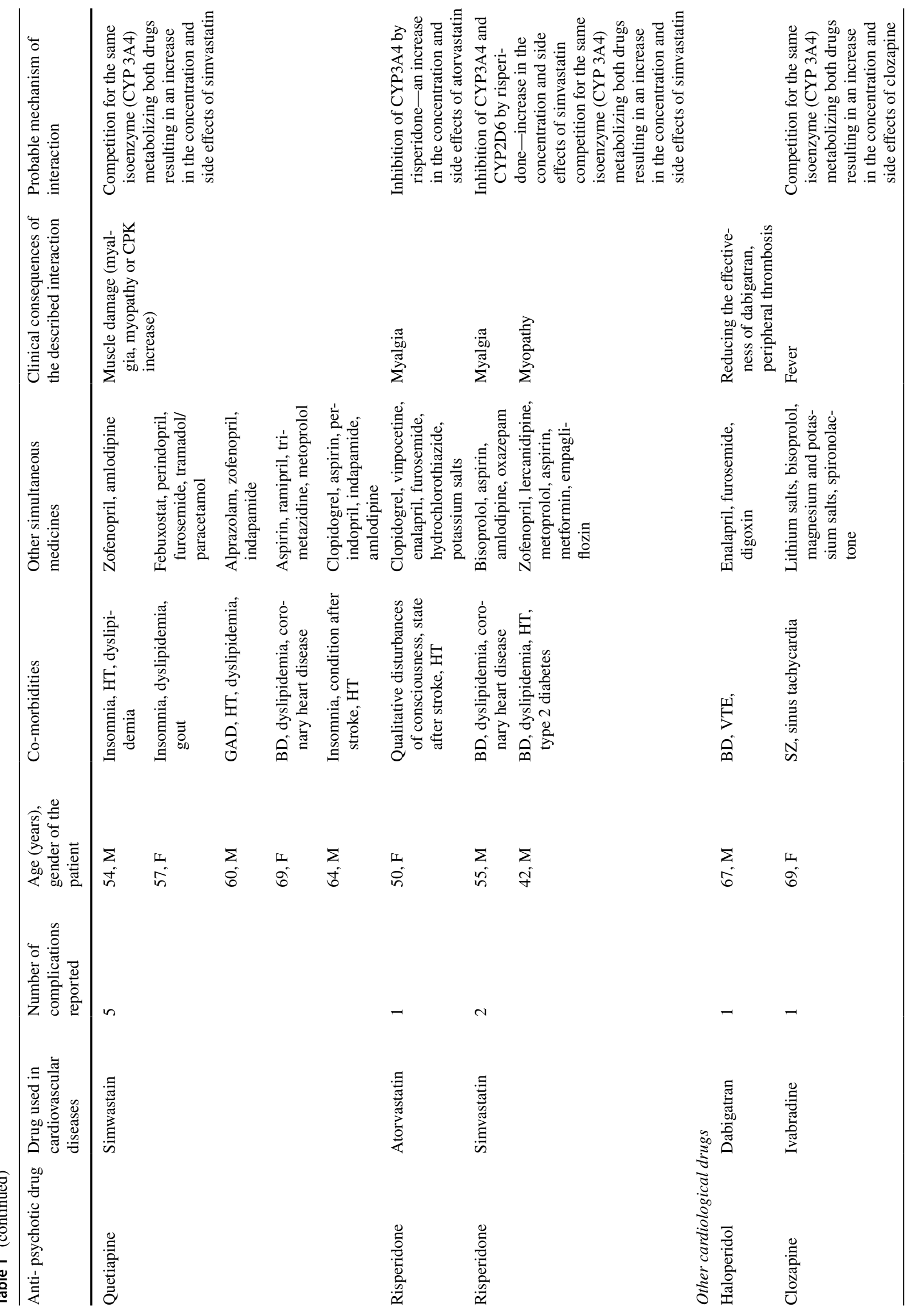




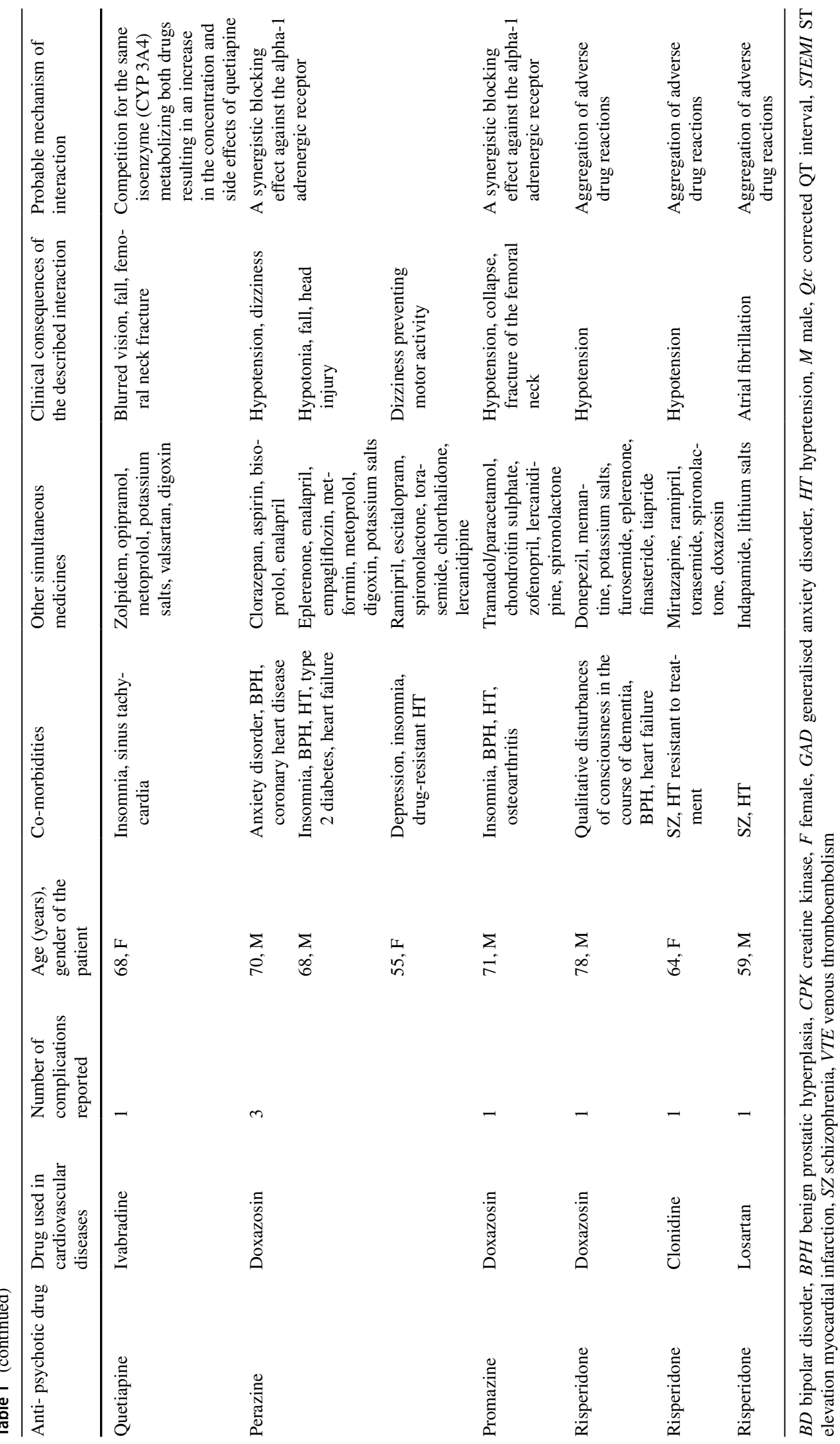


patient using aripiprazole and metoprol experienced a seizure. The mean age in the subgroup of patients using beta-blockers was 63.62 years $(\mathrm{SD}=5.87)$.

Another large group of interactions with antipsychotics concerned statins ( $n=12$, approx. 23\% of cases). In 11 cases, the effects of the interaction were muscle disorders, i.e., myalgia, myopathy, or creatine kinase. The interaction of atorvastatin with haloperidol $(n=1)$, quetiapine $(n=3)$, and risperidone $(n=1)$ and simvastatin with quetiapine $(n=5)$ and risperidone $(n=2)$ were observed. The average age in this group was 58.92 years $(\mathrm{SD}=8.41)$.

A total of 11 interactions were observed $(n=11$, approximately $21 \%$ ) in patients using LPP and anti-arrhythmic drugs such as amiodarone (class III anti-arrhythmic drug by Williams), flecainide, or propafenone (class Ic). In two patients using amiodarone with asenapine or ziprasidone, ventricular arrhythmias have been reported. In four cases, after combining olanzapine with propafenone, clozapine with amiodarone, and aripiprazole with amiodarone, akathisia was observed. Two people using clozapine with amiodarone or propafenone developed salivation.

Six cases related to combinations of drugs from the group of calcium channel blockers with antipsychotics ( $n=6$, approximately $11.5 \%$ of cases) - two interactions of verapamil, two diltiazem, and one for amlodipine and lercanidipine. In two cases, hypotonia was observed when combining haloperidol with amlodipine or lercanidipine. The interaction between risperidone and verapamil led to the occurrence of bradycardia, and between diltiazem and sertindole to ventricular arrhythmias.

The remaining ten cases of interactions related to cardiac drugs other than beta-blockers, calcium channel blockers, statins, and Williams class Ic or III anti-arrhythmics. In five patients, there was an interaction between doxazone and antipsychotics. In four cases, hypotonia was observed when doxazosin was combined with promazin, pernazine, or risperidone. One person who used perinazine with doxazosin had severe dizziness that prevented motor activity. One case of interaction between haloperidol and oral anticoagulant dabigatran was indicated, which resulted in decreased dabigatran efficacy and the occurrence of peripheral thrombosis. Two interactions were related to the use of ivabradine (a multifunctional medicine with selective blocking of the sinus-vestibular channel, used to treat ischemic heart disease)-fever occurred with combination with clozapine, and with quetiapineblurred vision and collapse resulting in fracture of the femoral neck. There has been hypotension observed in a person using risperidone with clonidine. The combination of risperidone with losartan caused in one of the analyzed cases atrial fibrillation. Table 2 presents the metabolism of selected antipsychotics by CYP 450 isoenzymes.
Table 2 Metabolism of selected antipsychotics by liver CYP450 isoenzymes (based on Refs. [12,13])

\begin{tabular}{ll}
\hline Antipsychotic & $\begin{array}{l}\text { CYP 450 isoenzymes for which antipsy- } \\
\text { chotic is a substrate }\end{array}$ \\
\hline Amisulpride & - \\
Aripiprazole & 3A4, 3A5, 3A7, 2D6 \\
Asenapine & 1A2, 3A4, 2D6 \\
Brexpiprazole & 3A4, 2D6 \\
Cariprazine & 3A4, 2D6 \\
Chlorprothixene & 2D6 \\
Clozapine & 1A2, 3A4, 2C19, 2D6 \\
Haloperidol & 1A2, 3A4, 3A5, 3A7, 2D6 \\
Lurasidone & 3A4 \\
Olanzapine & 1A2, 2D6 \\
Paliperidone & 3A4, 3A5, 2D6 \\
Perazine & $-1 \mathrm{~A} 2,3 \mathrm{~A} 4,2 \mathrm{C} 19$ \\
Perphenazine & $1 \mathrm{~A} 2,3 \mathrm{~A} 4,2 \mathrm{~B} 6,2 \mathrm{C} 8,2 \mathrm{C} 9,2 \mathrm{C} 18,2 \mathrm{C} 19,2 \mathrm{D} 6$ \\
Promazine & 1A2, 3A4, 2C9, 2C19, 2D6 \\
Quetiapine & 3A4, 3A5, 3A7, 2C19 \\
Risperidone & $3 \mathrm{~A} 4,3 \mathrm{~A} 5,3 \mathrm{~A} 7,2 \mathrm{D} 6$ \\
Sertindole & $3 \mathrm{~A} 4,2 \mathrm{D} 6$ \\
Sulpiride & - \\
Tiapryd & - \\
Ziprasidon & $3 \mathrm{~A} 4$ \\
Zuklopentiksol & $3 \mathrm{~A} 4,2 \mathrm{D} 6$ \\
\hline
\end{tabular}

\section{Discussion}

The highest number of interactions was observed in the case of antipsychotic connections with beta-blockers. Adverse effects were due to pharmacokinetic, pharmacodynamic, or aggregation of side effects. Metoprolol when used in combination with aripiprazole, clozapine, perphenazine, or sertindole significantly inhibited the metabolism of antipsychotics at CYP2D6 isoenzyme cytochrome P450. The effect was an increase in the concentration of antipsychotic in the blood followed by an increase in the risk of side effects and toxicity-the occurrence of increased salivation and lowering of the seizure threshold in the case of combination with aripiprazole, myoclonus, and urine retention for clozapine, bradycardia when used together with perphenazine, and ventricular arrhythmias when metoprolol combined with sertindole. Patients using concomitantly chlorprotixene with metoprolol or nebivolol showed hypotonia. The mechanism of these interactions most probably consisted in intensification of the hypotensive effect of beta-blockers by chlorprothixene-antipsychotic having an $\alpha$-blocking effect. The combination of another beta-blocker, atenolol, and risperidone caused one patient to develop atrial fibrillation-as a possible mechanism, the aggravation of the side effects of both drugs was identified. The analogous 
mechanism was probably responsible for the ventricular arrhythmias observed in three patients using ziprasidone and sotalol, which, in one case, resulted in death of the patient. So far, none of the above-mentioned interactions between beta-blockers and antipsychotics have been described in the literature.

Among the interactions of drugs from the group of calcium channel blockers (CCBs) with antipsychotics, there were two cases of haloperidol interactions in people using either amlodipine or lercanidipine. The source of these interactions was probably the inhibition of the metabolism of CCB by haloperidol-a drug that inhibits the activity of, among others, CYP 3A4 isoenzyme. Hence, the effect of the interaction was the severity of CCB activity and the occurrence of hypotension. There were two cases of interactions involving diltiazem. When this medicine was combined with quetiapine, the patient developed blurred vision, collapse with subsequent fracture of the femoral neck. In another patient, the interaction between diltiazem and sertindole was probably responsible for the occurrence of ventricular arrhythmias. For both of these interactions, the proposed mechanism is the diltiazem inhibitory effect on CYP 3A4 and, consequently, the fall in the metabolism of quetiapine and sertindole. The analogous mechanism most likely underlies another interactions described by us-verapamil with quetiapine. With high probability, inhibition of quetiapine metabolism by verapamil led to the patient's restless legs syndrome. In case of the next interaction of verapamil, this time with risperidone, it was likely that the side effects of both drugs were combined and bradycardia was induced.

In the anti-arrhythmic drug group belonging to the Ic and III groups according to Williams, there were six cases of interactions involving amiodarone. In case of the drug interaction with aripiprazole or clozapine, amiodarone inhibited that CYP 2D6 activity was likely and consequently antipsychotic metabolism was inhibited, resulting in extrapyramidal symptoms, akathisia, and tremor (for aripiprazole) and salivation, akathisia, tremor, hyperthermia (for clozapine). One case of a 75-year-old patient suffering from schizophrenia has been described so far, in which an increase of clozapine serum concentration of 6.5 times was observed after the addition of amiodarone to clozapine [14]. The patient denied the symptoms of excessive sedation, while he presented increased formal thinking disorders. We also described two interactions of amiodarone, the mechanism of which was probably the sum of side effects of drugs in the form of conductive disorders-the combination of this drug with ziprasidone or asenapine resulted in the occurrence of ventricular arrhythmias.

The observed interactions of propafenone probably resulted from inhibition of cytochrome $\mathrm{P} 450$ isoenzymes by this antiarrhythmic drug. The combination with clozapine resulted in the inhibition of this drug metabolism at the CYP1A2 level and the occurrence of intense salivation in the patient. We have described three interactions of propafenone with olanzapine, which seems to be supported by propafenone inhibition of CYP1A2 and CYP2D6 isoenzymes, which resulted in gynecomastia, galactorrhea, akathisia, and priapism. Another anti-arrhythmic drug which, in combination with olanzapine, caused one patient pancreatitis is flecainide. The inhibition of CYP2D6 isoenzyme by flecainide is also a probable mechanism here.

Among the interactions of antipsychotics and statins described by us, the majority was most likely caused by the effect of inhibiting statin metabolism or competition for the same isozyme metabolizing both drugs, and consequently escalating the level of serum statin and undesirable effects on the part of the muscular system. Drugs affecting the metabolism of atorvastatin were: haloperidol, quetiapine, risperidone, and simvastatin quetiapine and risperidone. Noteworthy is one case of interaction between atorvastatin and quetiapine, where the inhibitory effect of statin on CYP3A4 and quetiapine metabolism led to edema of the lower limbs. To date, one case of a 22-year-old schizophrenic patient has been described, in which the addition of simvastatin to risperidone therapy induced a high level of serum statin followed by rhabdomyolysis and a syndrome of the fascia compartments in the lower limb [15]. The authors concluded that the potential mechanism of interaction could be the competition of both drugs for the active site of the CYP3A4 isoenzyme, which participates in the metabolism of both drugs.

We described two cases of interaction with ivabradine, with quetiapine and clozapine, where most likely due to competition for the same isoenzyme (CYP3A4), the metabolism of antipsychotics was inhibited. This was associated with the occurrence of fever in a patient using clozapine and blurred vision, collapse, and consequent fracture of the femoral neck in a person taking quetiapine. Furthermore, summation of the alpha-blocker effect of doxazosin with pernazine, promazin, or risperidone was associated with the occurrence of hypotension, sometimes additionally with dizziness and falls. Similarly, in the case of combination of risperidone with clozapine, aggravation of side effects in one patient triggered hypotension. An analogous mechanism was probably responsible for the occurrence of atrial fibrillation in a patient treated with risperidone and losartan. It is worth noting that in addition to the two clinical cases mentioned in the discussion, the interactions of cardiac drugs with antipsychotics collected by us have not been described in the literature, to our knowledge.

\section{Conclusions}

As mentioned in the introduction, due to the co-existence of mental diseases such as bipolar disorder and schizophrenia with cardiovascular disease, the use of antipsychotic 
polytherapy with cardiac drugs is a frequent phenomenon. Based on our analysis, it can be concluded that while using cardiovascular medication with antipsychotics, particular attention should be paid to the high risk of interaction and the resulting side effects. Clinical decisions should be associated with the search for optimal, as safe as possible, drug combinations.

Acknowledgements Open access publishing of this article was funded by the Ministry of Science and Higher Education under the agreement No. 879/P-DUN/2019.

Author contributions The authors certify that professional language editing of the article has been performed. MS: conceptualization, formal analysis, methodology, investigation, data curation, writingoriginal draft, and writing - review and editing. JW: conceptualization, methodology, investigation, data curation, writing — original draft, writing - review and editing, supervision, and project administration. AG: formal analysis, writing-original draft, and writing-review and editing. JW: conceptualization, writing - review and editing, and supervision.

\section{Compliance with ethical standards}

Conflict of interest The authors declare no conflict of interest.

Open Access This article is licensed under a Creative Commons Attribution 4.0 International License, which permits use, sharing, adaptation, distribution and reproduction in any medium or format, as long as you give appropriate credit to the original author(s) and the source, provide a link to the Creative Commons licence, and indicate if changes were made. The images or other third party material in this article are included in the article's Creative Commons licence, unless indicated otherwise in a credit line to the material. If material is not included in the article's Creative Commons licence and your intended use is not permitted by statutory regulation or exceeds the permitted use, you will need to obtain permission directly from the copyright holder. To view a copy of this licence, visit http://creativecommons.org/licenses/by/4.0/.

\section{References}

1. Mathers C, Boerma T, Ma Fat D, World Health Organization. The global burden of disease: 2004 update. Geneva: World Health Organization; 2008.
2. De Hert M, Detraux J, Vancampfort D. The intriguing relationship between coronary heart disease and mental disorders. Dialogues Clin Neurosci. 2018;20(1):31-40.

3. De Hert M, Correll CU, Bobes J, Cetkovich-Bakmas M, Cohen $\mathrm{D}$, Asai I, et al. Physical illness in patients with severe mental disorders. I. Prevalence, impact of medications and disparities in health care. World Psychiatry. 2011;10(1):52-77.

4. Correll CU, Solmi M, Veronese N, Bortolato B, Rosson S, Santonastaso $\mathrm{P}$, et al. Prevalence, incidence and mortality from cardiovascular disease in patients with pooled and specific severe mental illness: a large-scale meta-analysis of 3,211,768 patients and 113,383,368 controls. World Psychiatry. 2017;16(2):163-80.

5. Fan Z, Wu Y, Shen J, Ji T, Zhan R. Schizophrenia and the risk of cardiovascular diseases: a meta-analysis of thirteen cohort studies. J Psychiatr Res. 2013;47(11):1549-56.

6. Hayes JF, Miles J, Walters K, King M, Osborn DP. A systematic review and meta-analysis of premature mortality in bipolar affective disorder. Acta Psychiatr Scand. 2015;131(6):417-25.

7. Goldstein BI, Fagiolini A, Houck P, Kupfer DJ. Cardiovascular disease and hypertension among adults with bipolar I disorder in the United States. Bipolar Disord. 2009;11(6):657-62.

8. Crump C, Sundquist K, Winkleby MA, Sundquist J. Comorbidities and mortality in bipolar disorder: a Swedish national cohort study. JAMA Psychiatry. 2013;70(9):931-9.

9. Masnoon N, Shakib S, Kalisch-ellett L, Caughey GE. What is polypharmacy? A systematic review of definitions. BMC Geriatr. 2017;17(1):230.

10. Kukreja S, Kalra G, Shah N, Shrivastava A. Polypharmacy in psychiatry: a review. Mens Sana Monogr. 2013;11(1):82-99.

11. Volpe M, Chin D, Paneni F. The challenge of polypharmacy in cardiovascular medicine. Fundam Clin Pharmacol. 2010;24(1):9-17.

12. Bazire S. Psychotropic drug directory 2018. Dorsington: LloydReinhold Communications; 2018.

13. Wishart DS, Feunang YD, Guo AC, Lo EJ, Marcu A, Grant JR, et al. DrugBank 5.0: a major update to the DrugBank database for 2018. Nucleic Acids Res. 2018;46(D1):D1074-D10821082.

14. Stevens JR, Freudenreich O, Stern TA. Elevated clozapine serum level after treatment with amiodarone. Psychosomatics. 2008;49(3):255-7.

15. Webber MA, Mahmud W, Lightfoot JD, Shekhar A. Rhabdomyolysis and compartment syndrome with coadministration of risperidone and simvastatin. J Psychopharmacol. 2004;18(3):432-4.

Publisher's Note Springer Nature remains neutral with regard to jurisdictional claims in published maps and institutional affiliations.. 